\section{Rezeptorexpression bei Brustkrebs nach neoadjuvanter Chemotherapie verändert}

\begin{abstract}
Eine pathologische Komplettremission ( $\mathrm{pCR}$ ) nach neoadjuvanter Chemotherapie (NAC) ist der wichtigste Prädiktor für den Therapieerfolg beim operablen, lokal fortgeschrittenen Mammakarzinom. Nach der NAC kann sich allerdings die Expression von Hormonrezeptoren und HER2 verändern.
\end{abstract}

$\mathrm{D}$ as zeigen Auswertungen von $\mathrm{pCR}$ und der Expression von Östrogenrezeptor (ER), Progesteronrezeptor (PgR) und HER2 bei 21.755 Patientinnen mit frühem Mammakarzinom im japanischen Brustkrebsregister, die sich einer NAC unterzogen hatten. Als pCR war dabei das Fehlen eines invasiven Tumors bei der Operation definiert. Die HER2-Überexpression wurde immunhistochemisch (IHC) und/oder mit Fluoreszenz-in-situHybridisierung (FISH) festgestellt. Eine pCR wiesen 5,7\% der Patientinnen mit luminalem Tumor auf $(\mathrm{n}=8.730), 24,6 \%$ derjenigen mit HER2-positivem Tumor $(\mathrm{n}=4.403)$ und $18,9 \%$ der Patientinnen mit triple-negativem Tumor $(\mathrm{n}=3.660)$. tiven Tumoren hatten ER-negative nach der NAC mit 31,6\% deutlich häufiger eine pCR als ER-positive (17,0\%). Von den Frauen mit HER2-positiven Tumoren, die Trastuzumab als neoadjuvante Therapie erhalten hatten $(n=2.437)$, erreichten $31,4 \%$ eine pCR. Wurde keine Trastuzumab-Therapie durchgeführt $(\mathrm{n}=1.966)$, lag die pCR-Rate nur bei 16,2\%. Die pCRRaten in den Subgruppen entsprechen denen in klinischen Studien.

$21,4 \%$ der Patientinnen mit initial HER2-positivem Tumor wiesen nach der neoadjuvanten Therapie keine HER2-Überexpression mehr auf. Umgekehrt fand sich bei 3,4\% der Frauen mit
Unter den Patientinnen mit HER2-posi- ursprünglich HER2-negativem Tumor nach der NAC ein HER2-positiver Befund. Auch der ER-Status änderte sich bei einigen Patientinnen: Von 10.973 vor NAC ER-positiven Tumoren waren 499 $(4,6 \%)$ anschließend ER-negativ, 519 von $5.607(9,3 \%)$ vor NAC ER-negativen Tumoren waren anschließend ER-positiv. Von 8.280 PgR-positiven Tumoren waren nach NAC außerdem 1.545 (18,7\%) negativ, von 8.235 initial PgR-negativen Tumoren 766 (9,3\%) PgR-positiv.

Fazit: Die Registerdaten belegen eindrücklich die Diskordanz der Expressionsanalysen vor und nach NAC und zeigen damit die Notwendigkeit einer erneuten Testung von Hormonrezeptorund HER2-Expression nach neoadjuvanter Therapie, um die bestmögliche Folgetherapie zu bestimmen.

Friederike Klein

Niikura $\mathrm{N}$ et al. Changes in tumor expression of HER2 and hormone receptors status after neoadjuvant chemotherapy in 21755 patients from the Japanese breast cancer registry. Ann Oncol. 2016;27(3):480-7.

\title{
Nach neoadjuvanter Brustkrebstherapie noch bestrahlen?
}

Die Strahlentherapie nach Mastektomie (PMRT) führt bei positivem Lymphknotenbefund zu einem besseren Überleben. Die Bestrahlung der regionären Lymphknoten (RNI) nach brusterhaltender Operation oder Mastektomie verlängert das krankheitsfreie Überleben. Nun wurde der Einfluss von PMRT bzw. RNI nach neoadjuvanter Chemotherapie (NAC) untersucht.

\footnotetext{
$\mathrm{n}$ der nationalen Krebsdatenbank der I USA wurden 15.315 Frauen mit Mammakarzinom cT1-3cN1 M0 identifiziert, die zwischen 2003 und 2011 eine NAC erhalten hatten.

Differenziert nach Operationsmethode (Mastektomie versus brusterhaltende Therapie, BET) und Nodalstatus nach NAC (ypN0 versus ypN+) wurden 4 Gruppen gebildet:

-Mast-ypN0: Mastektomie, kein pathologischer Lymphknotenbefall im Resektat $(\mathrm{n}=3.040)$

-Mast-ypN+: Mastektomie, positiver Lymphknotenstatus $(\mathrm{n}=7.243)$

-BET-ypN0: BET, nodal-negativ $(\mathrm{n}=$ 2.070)
}

- BET-ypN+: BET, positiver Lymphknotenstatus $(\mathrm{n}=2.962)$.

Die univariate Analyse wies auf eine Assoziation von PMRT und verbessertem Gesamtüberleben bei Mast-ypNo ( $\mathrm{p}=$ $0,019)$ sowie bei Mast-ypN+ $(\mathrm{p}<0,001)$ hin. Wurden in der multivariaten Analyse Faktoren wie Alter, Komorbidität, cT-Stadium, pathologische Komplettremission, Axilladissektion, yPN- und Östrogenrezeptorstatus sowie endokrine Therapie einbezogen, blieb die PMRT in beiden Mastektomie-Gruppen unabhängig davon mit einem längeren Überleben assoziiert. Für Patientinnen mit MastypN0 lag die Hazard Ratio (HR) bei 0,729 (95\%-Konfidenzintervall [95\%-KI]
0,566-0,939; $\mathrm{p}=0,015)$, für Patientinnen mit Mast-ypN+ bei 0,772 (95\%-KI 0,689$0,866$; $p<0,001)$. In einer Subgruppenanalyse war die PMRT unabhängig vom Nodalstatus nach Mastektomie (ypN0, ypN1, ypN2-3) von Vorteil (jeweils $\mathrm{p}<$ $0,05)$. Eine RNI zusätzlich zur StandardStrahlentherapie nach BET führte unabhängig vom Nodalstatus nach NAC nicht zu einem Überlebensvorteil.

Fazit: Diese bisher größte Analyse zur Strahlentherapie bei cN1-Mammakarzinom nach NAC belegt einen Überlebensvorteil für die PMRT nach Mastektomie unabhängig vom Nodalstatus, nicht aber für die zusätzliche RNI nach BET.

Friederike Klein

Rusthofen CG et al. The impact of postmastectomy and regional nodal radiation after neoadjuvant chemotherapy for clinically lymph node-positive breast cancer: a National Cancer Database (NCDB) analysis. Ann Oncol. 2016;27(5):818-27. 